\title{
Does Reusing Pre-trained NLP Model Propagate Bugs?
}

\author{
Mohna Chakraborty \\ mohnac@iastate.edu \\ Dept. of Computer Science, Iowa State University \\ Ames, Iowa, USA
}

\begin{abstract}
In this digital era, the textual content has become a seemingly ubiquitous part of our life. Natural Language Processing (NLP) empowers machines to comprehend the intricacies of textual data and eases human-computer interaction. Advancement in language modeling, continual learning, availability of a large amount of linguistic data, and large-scale computational power have made it feasible to train models for downstream tasks related to text analysis, including safety-critical ones, e.g., medical, airlines, etc. Compared to other deep learning (DL) models, NLP-based models are widely reused for various tasks. However, the reuse of pre-trained models in a new setting is still a complex task due to the limitations of the training dataset, model structure, specification, usage, etc. With this motivation, we study BERT, a vastly used language model (LM), from the direction of reusing in the code. We mined 80 posts from Stack Overflow related to BERT and found 4 types of bugs observed in clients' code. Our results show that $13.75 \%$ are fairness, $28.75 \%$ are parameter, $15 \%$ are token, and $16.25 \%$ are version-related bugs.
\end{abstract}

\section{CCS CONCEPTS}

- Software and its engineering $\rightarrow$ Software defect analysis; • Computing methodologies $\rightarrow$ Machine learning.

\section{KEYWORDS}

Bug, Deep Learning, NLP, BERT, Reuse

\section{ACM Reference Format:}

Mohna Chakraborty. 2021. Does Reusing Pre-trained NLP Model Propagate Bugs?. In Proceedings of the 29th ACM Joint European Software Engineering Conference and Symposium on the Foundations of Software Engineering (ESEC/FSE '21), August 23-28, 2021, Athens, Greece. ACM, New York, NY, USA, 3 pages. https://doi.org/10.1145/3468264.3473494

\section{RESEARCH PROBLEM AND MOTIVATION}

NLP is a sub-domain of computer science that studies how a machine can understand or interpret text or speech to perform functional tasks, e.g., information extraction [19, 40], sentiment analysis $[17,39]$, question-answering [38], and others. These tasks can be done either in a single [37], or multi-task [6] framework. We can always train these task-based models from scratch. However, a more efficient way is to reuse the existing pre-trained models. In figure 1, a language model, BERT, pre-trained on Wikipedia pages

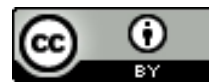

This work is licensed under a Creative Commons Attribution International 4.0 License. ESEC/FSE '21, August 23-28, 2021, Athens, Greece

(c) 2021 Copyright held by the owner/author(s).

ACM ISBN 978-1-4503-8562-6/21/08.

https://doi.org/10.1145/3468264.3473494 and book corpus, has been reused to perform various tasks by training on a corpus and further fine-tuning the hyperparameters or using task-specific datasets by a user. Thus, instead of creating a model from scratch, these fine tuned models' can be reproduced, saving resource exhaustion and carbon footprint [32,36].

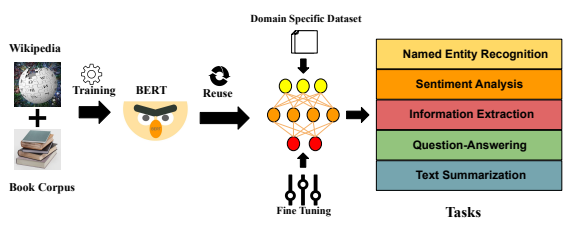

Figure 1: Reusing a language model for different tasks

Reuse of the LM is akin to software code cloning [16, 25, 34], where a piece of code is reused or cloned to perform specific tasks. While code cloning has helped in the software development process, it often introduces bugs without the users' knowledge. Similarly, we can consider LM as a single piece of code, and reusing these blackbox models facilitates bugs in users' code, if incorrectly used. Based on the same motivation, we study BERT [7], a well-known and wellversed LM, trained on a total of 340M parameters and 16GB data. We empirically observed that the bugs in the client code are due to limitations posed by the model or improper usage of the model by manually going through 80 posts from Stack Overflow. To this end, we identified the key issues responsible for such bugs in users' code. The key findings of this paper are: a) Depending on the training corpus, LM is susceptible to learn intrinsic bias. b) Improper usage of the special tokens in clients' code can facilitate bugs. c) Efficient selection criteria of hyperparameters (e.g., batch size) helps the LM to learn word embedding better. d) Implementation with incorrect versions of the models' frameworks causes bugs in the program.

\section{RELATED WORK}

Our closest works are broadly classified into two categories.

Study on Software Bug Propagation: There are multiple benefits of cloning a piece of code; however, it facilitates bugs transmission to other code fragments [2, 3, 10, 11, 14, 15, 31]. While study of the characteristics of the bugs [33], localizing [21, 24], and fixing them [1] have been done extensively. They are done on traditional software, which is mostly white-box in nature. Whereas, an NLP-based software is a black-box monolithic system.

Study on Deep Neural Network Bug: Zhang et al. [41] studied the bugs faced by the developers while using the Tensorflow framework and categorized them into causes and symptoms. Islam et al. [12] proposed a classification adapting traditional software bug types, root cause [4], bug fixes [13] and impacts to understand the bugs found in 5 different DL-based frameworks. While these studies 
have charted the course in DL bugs, the types of bugs observed by developers while using pre-trained LM has not been studied.

\section{METHODOLOGY AND FINDINGS}

Methodology: First, we collect the data that includes pre-trained model usages, mine issues from Stack Overflow, created the classification scheme, and finally, manually labeled the bugs. Here, we focus on the transformer-based LM [35]. Among those models $[18,20,22,23]$, we choose to study BERT since it has the highest number of citations and GitHub star-count (18,745 citations and $27.9 \mathrm{~K}$ star count). In order to identify the bugs faced by developers while reusing BERT, we focus on the issues reported by developers in the open-source Q\&A forum, Stack Overflow, from March 2019 to January 2021, by manually verifying the posts that have code snippet, score $\geq 2$ and title or the body having any of the keywords "BERT" or "bert-language-model" to ensure the quality. Based on the selection criteria, we found 80 Stack Overflow posts from 141 posts and labeled them as Yes (if the bug is present) or No (Otherwise).

Findings: We observed that $26.25 \%$ of the posts do not impact the downstream tasks. $13.75 \%$ of the posts refer to bugs caused due to biased behavior of the model, $15 \%$ of them are due to misuse of the additional tokens, $28.75 \%$ of the bugs are due to wrong parameter choice, and $16.25 \%$ of them are caused due to version confusion.

a.) Fairness: In machine learning, fairness deals with ensuring how bias in the data and model does not lead to an unfavorable decision based on certain features. For instance, Google Perspective API was found to generate a high toxicity score for sentences involving a particular section of the population [5, 8]. While investigating, it was seen that those representative words were infrequent, and they belonged to a shorter sentence in the corpus. The quality and quantity of data available for training a LM introduce bias in its performance unknowingly. For instance, Multilingual BERT (mBERT) [9], a variant of BERT trained over 104 languages, favors few languages more based on the uneven distribution of different languages in the training corpus rather than having a language-neutral understanding. The Stack Overflow post [26] discusses the biased behavior of the model for the Khmer language. Another example, if we have 2 texts in Chinese language, one of them composed of 2 words, $\mathrm{n}=$ $w_{1} w_{2}=a_{1} a_{2} a_{3} a_{4}$, where $w_{1}$ is composed of 2 alphabets $a_{1} a_{2}$ and $w_{2}$ composed of $a_{3} a_{4}$. The other text composed of 4 alphabets like $a_{1} a_{2} a_{3} a_{4}$. Since the Chinese language is character-tokenized and does not have a white-space character between the words, thus the embedding of both texts will be the same, which is not desired. Even if the developers introduce spaces around every character, the embedding will be the same for different contextualized text.

b.) Special Tokens: During pre-processing of input text by LM, several additional tokens are added. For BERT, tokens used are [CLS], [SEP], [PAD], and [UNK]. Each token has its functionality, e.g., [CLS], classification token, is prepended to the sentence. It is responsible for capturing the context of the sentence. [SEP] is a token which is appended to the sentences to separate them. BERT performs the tokenization by splitting the input text based on words, characters, and symbols defined as per the vocabulary on which BERT's tokenizer is trained. This Stack Overflow post [29] shows an issue when we add tokens that are not pre-defined in BERT's vocabulary. When a word does not exist in the vocabulary, the tokenizer will further chunk it into sub-words or character tokens per BERT's vocabulary. For example, "chowmein" will be tokenized into ['chow'], ['me'], ['in']. Since such words are rare and limited, though the model will learn an embedding, however it will misrepresent its context as per the neighboring words. Therefore, the distorted meaning will propagate through the model's layers, and thus the semantics of the entire text will be lost. Further research is needed to understand the usage and improvement of special tokens in the LM.

c.) Model Parameter: For optimal performance, different finetuning methods are adopted: 1) Train the entire model on a dataset and feed the output to a softmax layer. In this case, the error is backpropagated through the entire architecture, and the pre-trained weights are updated. 2) Keep the weights of lower layers of the model frozen while retraining only the higher layers. 3) Freeze all the layers, add a few neural network layers and train the model. Thus, only the weight of the new layers are updated during training. Post [30] from Stack Overflow, where BERT gives random output each time due to the random initialization of the classifier layer. Another post [27] where the output is not deterministic, i.e., for the same input, BERT gives different results. Therefore, finalizing ideal set of parameters is an extensive task but an optimal set will speed up the gradient descent. Similarly, allowing a model to train for more epochs increases the risk of overfitting. However, if the parameters are changed after every epoch, it may be counterproductive as the model is trained only for few epochs. Therefore, customizing parameters' value will help in resource optimizing. Another example of the effect of the parameter is when the average length of the sentences in a dataset is more than the sequence length; the model may not learn the reference. Sequence length refers to the length of the sentence that the model can process. Assuming the max length is 4 , and the input text is 'The staff works in the hospital $24^{*} 7$ '. Considering parameter restriction, the first batch of the input will be ['The'], ['staff'], ['works'], ['in'] and the second batch will be , ['the'], ['hospital'], ['24*7']. Here, neither of the batches capture relevant context.

d.) Model Version: Sometimes, developers employ pre-trained models unaware that the version has been updated and encounter incompatible bugs among the APIs. This issue is caused because of not updating the documentation of the model concerning the new release and not because of unawareness among the developers. Post [28] from Stack Overflow, where the developer was unaware that the pre-trained BERT does not return tuples in 3.x release of the transformers library and ended up with an unexpected result.

\section{CONCLUSION}

In this work, we study one of the most popular NLP-based models, BERT, to understand the bugs encountered by developers while reusing it. We have empirically evaluated bugs from Stack Overflow and identified four common bug types in the model.

\section{ACKNOWLEDGEMENT}

This research has been done under the supervision of Dr. Hridesh Rajan and the guidance of fellow lab mates Rangeet Pan and Sumon Biswas. We thank all reviewers for their constructive comments. 


\section{REFERENCES}

[1] Andrea Arcuri and Xin Yao. 2008. A novel co-evolutionary approach to automatic software bug fixing. In 2008 IEEE Congress on Evolutionary Computation (IEEE World Congress on Computational Intelligence). IEEE, 162-168. https://doi.org/10. 1109/CEC.2008.4630793

[2] Lerina Aversano, Luigi Cerulo, and Massimiliano Di Penta. 2007. How clones are maintained: An empirical study. In 11th European Conference on Software Maintenance and Reengineering (CSMR'07). IEEE, 81-90. https://doi.org/10.1109/ CSMR.2007.26

[3] Liliane Barbour, Foutse Khomh, and Ying Zou. 2011. Late propagation in software clones. In 2011 27th IEEE International Conference on Software Maintenance (ICSM). IEEE, 273-282. https://doi.org/10.1109/ICSM.2011.6080794

[4] Boris Beizer. 1984. Software system testing and quality assurance. Van Nostrand Reinhold Co.

[5] Emily M Bender, Timnit Gebru, Angelina McMillan-Major, and Shmargaret Shmitchell. 2021. On the Dangers of Stochastic Parrots: Can Language Models Be Too Big?. In Proceedings of the 2021 ACM Conference on Fairness, Accountability, and Transparency. 610-623. https://doi.org/10.1145/3442188.3445922

[6] Zehui Dai, Cheng Peng, Huajie Chen, and Yadong Ding. 2020. A Multi-Task Incremental Learning Framework with Category Name Embedding for AspectCategory Sentiment Analysis. arXiv preprint arXiv:2010.02784 (2020).

[7] Jacob Devlin, Ming-Wei Chang, Kenton Lee, and Kristina Toutanova. 2018. Bert: Pre-training of deep bidirectional transformers for language understanding. arXiv preprint arXiv:1810.04805 (2018).

[8] Lucas Dixon, John Li, Jeffrey Sorensen, Nithum Thain, and Lucy Vasserman. 2018. Measuring and mitigating unintended bias in text classification. In Proceedings of the 2018 AAAI/ACM Conference on AI, Ethics, and Society. 67-73. https: //doi.org/10.1145/3278721.3278729

[9] GitHub mBERT Repository 2019. mBERT. https://github.com/google-research/ bert/blob/master/multilingual.md.

[10] Nils Göde and Rainer Koschke. 2011. Frequency and risks of changes to clones. In Proceedings of the 33rd International Conference on Software Engineering. 311-320. https://doi.org/10.1145/1985793.1985836

[11] Keisuke Hotta, Yukiko Sano, Yoshiki Higo, and Shinji Kusumoto. 2010. Is duplicate code more frequently modified than non-duplicate code in software evolution? An empirical study on open source software. In Proceedings of the foint ERCIM Workshop on Software Evolution (EVOL) and International Workshop on Principles of Software Evolution (IWPSE). 73-82. https://doi.org/10.1145/1862372.1862390

[12] Md Johirul Islam, Giang Nguyen, Rangeet Pan, and Hridesh Rajan. 2019. A comprehensive study on deep learning bug characteristics. In Proceedings of the 2019 27th ACM Joint Meeting on European Software Engineering Conference and Symposium on the Foundations of Software Engineering. 510-520. https: //doi.org/10.1145/3338906.3338955

[13] Md Johirul Islam, Rangeet Pan, Giang Nguyen, and Hridesh Rajan. 2020. Repairing deep neural networks: Fix patterns and challenges. In 2020 IEEE/ACM 42nd International Conference on Software Engineering (ICSE). IEEE, 1135-1146.

[14] Lingxiao Jiang, Zhendong Su, and Edwin Chiu. 2007. Context-based detection of clone-related bugs. In Proceedings of the the 6th joint meeting of the European software engineering conference and the ACM SIGSOFT symposium on The foundations of software engineering. 55-64. https://doi.org/10.1145/1287624.1287634

[15] Elmar Juergens, Florian Deissenboeck, Benjamin Hummel, and Stefan Wagner. 2009. Do code clones matter?. In 2009 IEEE 31st International Conference on Software Engineering. IEEE, 485-495. https://doi.org/10.1109/ICSE.2009.5070547

[16] Toshihiro Kamiya, Shinji Kusumoto, and Katsuro Inoue. 2002. CCFinder: A multilinguistic token-based code clone detection system for large scale source code. IEEE Transactions on Software Engineering 28, 7 (2002), 654-670. https: //doi.org/10.1109/TSE.2002.1019480

[17] Pei Ke, Haozhe Ji, Siyang Liu, Xiaoyan Zhu, and Minlie Huang. 2020. Sentilare: Linguistic knowledge enhanced language representation for sentiment analysis. In Proceedings of the 2020 Conference on Empirical Methods in Natural Language Processing (EMNLP). 6975-6988.

[18] Mike Lewis, Yinhan Liu, Naman Goyal, Marjan Ghazvininejad, Abdelrahman Mohamed, Omer Levy, Ves Stoyanov, and Luke Zettlemoyer. 2019. Bart: Denoising sequence-to-sequence pre-training for natural language generation, translation, and comprehension. arXiv preprint arXiv:1910.13461 (2019).

[19] Ying Lin, Heng Ji, Fei Huang, and Lingfei Wu. 2020. A joint neural model for information extraction with global features. In Proceedings of the 58th Annual Meeting of the Association for Computational Linguistics. 7999-8009. https: //doi.org/10.18653/v1/2020.acl-main.713
[20] Yinhan Liu, Myle Ott, Naman Goyal, Jingfei Du, Mandar Joshi, Danqi Chen, Omer Levy, Mike Lewis, Luke Zettlemoyer, and Veselin Stoyanov. 2019. Roberta: A robustly optimized bert pretraining approach. arXiv preprint arXiv:1907.11692 (2019).

[21] Stacy K Lukins, Nicholas A Kraft, and Letha H Etzkorn. 2008. Source code retrieval for bug localization using latent dirichlet allocation. In 2008 15th Working Conference on Reverse Engineering. IEEE, 155-164. https://doi.org/10.1109/WCRE. 2008.33

[22] Alec Radford, Jeffrey Wu, Rewon Child, David Luan, Dario Amodei, and Ilya Sutskever. 2019. Language models are unsupervised multitask learners. OpenAI blog 1, 8 (2019), 9.

[23] Colin Raffel, Noam Shazeer, Adam Roberts, Katherine Lee, Sharan Narang, Michael Matena, Yanqi Zhou, Wei Li, and Peter J Liu. 2019. Exploring the limits of transfer learning with a unified text-to-text transformer. arXiv preprint arXiv:1910.10683 (2019).

[24] Shivani Rao and Avinash Kak. 2011. Retrieval from software libraries for bug localization: a comparative study of generic and composite text models. In Proceedings of the 8th Working Conference on Mining Software Repositories. 43-52. https://doi.org/10.1145/1985441.1985451

[25] Chanchal K Roy, James R Cordy, and Rainer Koschke. 2009. Comparison and evaluation of code clone detection techniques and tools: A qualitative approach. Science of computer programming 74, 7 (2009), 470-495. https://doi.org/10.1016/j. scico.2009.02.007

[26] Stack Overflow 2019. Fairness Issue. https://stackoverflow.com/questions/ 59066593/can-i-pre-trained-bert-model-from-scratch-using-tokenized-inputfile-and-custom.

[27] Stack Overflow 2019. Not deterministic output. https://stackoverflow.com/ questions/56639938/bert-output-not- deterministic.

[28] Stack Overflow 2020. Model Version Issue. https://stackoverflow.com/questions/ 65132144/bertmodel-transformers-outputs-string-instead-of-tensor.

[29] Stack Overflow 2020. Token Issue. https://stackoverflow.com/questions/60068129/ transformers-pretrainedtokenizer-add-tokens-functionality.

[30] Stack Overflow 2021. Random output. https://stackoverflow.com/questions/ 65676389/huggingface-tfbertforsequenceclassification-always-predicts-thesame-label.

[31] Daniela Steidl and Nils Göde. 2013. Feature-based detection of bugs in clones. In 2013 7th International Workshop on Software Clones (IWSC). IEEE, 76-82. https: //doi.org/10.1109/IWSC.2013.6613047

[32] Emma Strubell, Ananya Ganesh, and Andrew McCallum. 2019. Energy and policy considerations for deep learning in NLP. arXiv preprint arXiv:1906.02243 (2019).

[33] Lin Tan, Chen Liu, Zhenmin Li, Xuanhui Wang, Yuanyuan Zhou, and Chengxiang Zhai. 2014. Bug characteristics in open source software. Empirical software engineering 19, 6 (2014), 1665-1705.

[34] Suresh Thummalapenta, Luigi Cerulo, Lerina Aversano, and Massimiliano Di Penta. 2010. An empirical study on the maintenance of source code clones. Empirical Software Engineering 15, 1 (2010), 1-34.

[35] Ashish Vaswani, Noam Shazeer, Niki Parmar, Jakob Uszkoreit, Llion Jones, Aidan N Gomez, Łukasz Kaiser, and Illia Polosukhin. 2017. Attention is all you need. In Advances in neural information processing systems. 5998-6008.

[36] Thomas Wiedmann and Jan Minx. 2008. A definition of 'carbon footprint'. Ecological economics research trends 1 (2008), 1-11.

[37] Hu Xu, Bing Liu, Lei Shu, and Philip S Yu. 2018. Double embeddings and cnnbased sequence labeling for aspect extraction. arXiv preprint arXiv:1805.04601 (2018).

[38] $\mathrm{Hu}$ Xu, Bing Liu, Lei Shu, and Philip S Yu. 2019. BERT post-training for review reading comprehension and aspect-based sentiment analysis. arXiv preprint arXiv:1904.02232 (2019)

[39] Ali Yadollahi, Ameneh Gholipour Shahraki, and Osmar R Zaiane. 2017. Current state of text sentiment analysis from opinion to emotion mining. ACM Computing Surveys (CSUR) 50, 2 (2017), 1-33. https://doi.org/10.1145/3057270

[40] Peng Zhang, Yunlu Xu, Zhanzhan Cheng, Shiliang Pu, Jing Lu, Liang Qiao, Yi Niu, and Fei Wu. 2020. TRIE: End-to-End Text Reading and Information Extraction for Document Understanding. In Proceedings of the 28th ACM International Conference on Multimedia. 1413-1422. https://doi.org/10.1145/3394171.3413900

[41] Yuhao Zhang, Yifan Chen, Shing-Chi Cheung, Yingfei Xiong, and Lu Zhang. 2018. An empirical study on TensorFlow program bugs. In Proceedings of the 27th ACM SIGSOFT International Symposium on Software Testing and Analysis. 129-140. https://doi.org/10.1145/3213846.3213866 